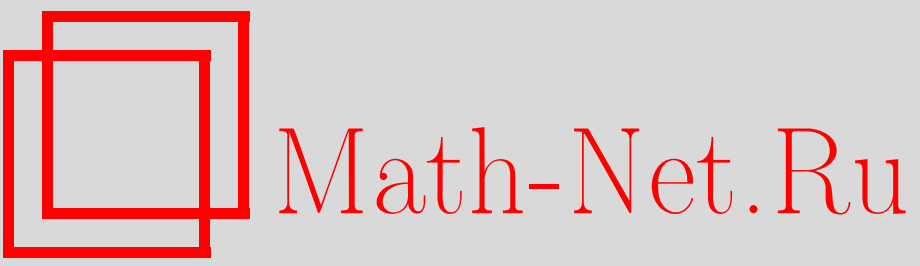

В. В. Абрамов, О ветвлении большого периодического решения системы дифференциальных уравнений с параметром, Итоги науки и техн. Сер. Соврем. мат. и ее прил. Темат. обз., 2019, том 168, 3-8

DOI: https://doi.org/10.36535/0233-6723-2019-168-3-8

Использование Общероссийского математического портала Math-Net.Ru подразумевает, что вы прочитали и согласны с пользовательским соглашением

http: //www. mathnet.ru/rus/agreement

Параметры загрузки:

IP : 54.147 .182 .235

26 апреля 2023 г., 12:53:08 


\title{
О ВЕТВЛЕНИИ БОЛЬШОГО ПЕРИОДИЧЕСКОГО РЕШЕНИЯ СИСТЕМЫ ДИФФЕРЕНЦИАЛЬНЫХ УРАВНЕНИЙ С ПАРАМЕТРОМ
}

\author{
(C) 2019 г. $\quad$ B. B. АБРАМОВ
}

\begin{abstract}
АннотАция. Исследована нормальная периодическая система обыкновенных дифференциальных уравнений с малым параметром, являющаяся квазилинейной в окрестности бесконечности. Предполагается, что правая часть системы имеет критическое линейное приближение. В терминах свойств первого однородного нелинейного приближения оператора монодромии получены условия существования периодического решения, у которого начальное значение является бесконечно большим при бесконечно малом значении параметра.
\end{abstract}

Ключевые слова: дифференциальное уравнение, периодическое решение, малый параметр, оператор монодромии.

\section{ON THE BRANCHING OF A LARGE PERIODIC SOLUTION}

\section{OF A SYSTEM OF DIFFERENTIAL EQUATIONS WITH A PARAMETER}

\author{
(c) 2019 V. V. ABRAMOV
}

\begin{abstract}
We study a normal periodic system of ordinary differential equations with a small parameter, which is quasilinear in a neighborhood of infinity, under the assumption that the right-hand side of the system has a critical linear approximation. In terms of the properties of the first homogeneous nonlinear approximation of the monodromy operator, we obtain conditions for the existence of a periodic solution whose initial value is infinitely large for an infinitesimal value of the parameter.
\end{abstract}

Keywords and phrases: differential equation, periodic solution, small parameter, monodromy operator.

AMS Subject Classification: $34 \mathrm{C} 25$

1. Введение. В монографии М. А. Красносельского [3] на основе свойств мажорирующей системы установлен признак существования периодического решения системы обыкновенных дифференциальных уравнений с некритической линейной частью, выделенной в окрестности бесконечности. В данной работе рассмотрим критический случай существования периодического решения системы указанного типа, предполагая, что правая часть системы зависит от малого параметра.

Рассмотрим систему дифференциальных уравнений вида

$$
\dot{x}=A(t) x+f(t, x, \mu),
$$

в которой $x \in \mathbb{R}^{n}, \mu \in \mathbb{R}^{m}$ - малый параметр, правая часть является $\omega$-периодической интегрируемой по $t$ при всех $x$ и $\mu$, функция $f(t, x, \mu)$ гладко зависит от фазовой переменной в окрестности бесконечности и от малого параметра, $\lim _{\alpha \rightarrow+\infty} \alpha^{-1}\|f(t, \alpha x, \mu / \alpha)\| \equiv 0$.

Обозначим через $x(t, a, \mu)$ решение с начальным значением $x(0, a, \mu)=a$. 
Определение 1. Решение $x\left(t, a^{*}, \mu^{*}\right)$ системы (1) называется большим, если существует параметризация $a^{*}=a(\alpha), \mu^{*}=\mu(\alpha), \Delta<\alpha$, при которой

$$
\lim _{\alpha \rightarrow+\infty} a^{*}=\infty, \quad \lim _{\alpha \rightarrow+\infty} \mu^{*}=0_{m} .
$$

Пара $\left(a^{\prime}(+\infty), \mu^{\prime}(+\infty)\right)$ задает направление ветвления решения от бесконечности.

Найдем условия существования большого $\omega$-периодического решения системы (1). С этой целью будем использовать схему рассуждений, предложенную в $[1,2]$.

2. Основные результаты. Так как при $x \rightarrow \infty$ и $\mu \rightarrow 0_{m}$ правая часть системы (1) близка к линейной, то при достаточно малых значениях параметра решения с большими по норме начальными значениями определены при $t \in[0, \omega]$. Поэтому с помощью оператора монодромии $a \rightarrow x(\omega, a, \mu)$ можно определить сдвиг начальной точки на период. Установим структуру оператора монодромии системы (1) в окрестности бесконечности фазового пространства.

Пусть $X(t)$ - фундаментальная матрица линейной системы $\dot{x}=A(t) x, X(0)=E$. Решение системы (1) представимо в виде $x(t, a, \mu)=X(t) a+y(t, a, \mu)$, где решение линейной системы $\dot{x}=A(t) x+f(t, x(t, a, \mu), \mu)$ имеет вид

$$
y(t, a, \mu)=X(t) \int_{0}^{t} X^{-1}(\tau) f(\tau, x(\tau, a, \mu), \mu) d \tau .
$$

По свойству системы (1) имеем $f_{x}^{\prime}\left(t, \infty, 0_{m}\right)=0_{n n}$. Из [3, теорема 2.2] следует, что $X(t)=$ $x_{a}^{\prime}\left(t, \infty, 0_{m}\right)$. Поэтому

$$
y_{a}^{\prime}\left(t, \infty, 0_{m}\right)=X(t) \int_{0}^{t} X^{-1}(\tau) f_{x}^{\prime}\left(\tau, \infty, 0_{m}\right) x_{a}^{\prime}\left(\tau, \infty, 0_{m}\right) d \tau=X(t) \int_{0}^{t} X^{-1}(\tau) \cdot 0_{n n} \cdot X(\tau) d \tau=0_{n n}
$$

т.е. $\lim _{\alpha \rightarrow+\infty} \alpha^{-1}\|y(t, \alpha a, \mu / \alpha)\| \equiv 0$.

Без ограничения общности рассуждений далее будем предполагать, что имеет место равенство $y(\omega, \alpha, \mu)=p(a, \mu)+\varphi(a, \mu)$, в котором $p(a, \mu)$ - главная нелинейная однородная часть оператора монодромии, $p(\alpha a, \mu / \alpha)=S(\alpha) p(a, \mu), S(\alpha)=\operatorname{diag}\left(\alpha^{k_{1}}, \ldots, \alpha^{k_{n}}\right), 0<k_{i}<1, i=\overline{1, n}$, $\lim _{\alpha \rightarrow+\infty}\left\|S^{-1}(\alpha) \varphi(\alpha a, \mu / \alpha)\right\| \equiv 0$. Тогда оператор монодромии системы (1) в окрестности бесконечности фазового пространства имеет вид

$$
x(\omega, a, \mu)=X a+p(a, \mu)+\varphi(a, \mu),
$$

где $X=X(\omega)$ - матрица монодромии.

Допустим, что имеет место некритический случай $\operatorname{det}(X-E) \neq 0$. Произвольно выберем для пары $(a, \mu)$ совместную параметриизацию $a^{*}=\alpha\left(a_{0}+\bar{a}(\alpha)\right), \mu^{*}=\alpha^{-1}\left(\mu_{0}+\bar{\mu}(\alpha)\right)$, в которой $\alpha>0$, $a_{0} \neq 0_{n}, \lim _{\alpha \rightarrow+\infty} \bar{a}(\alpha)=0_{n}, \lim _{\alpha \rightarrow+\infty} \bar{\mu}(\alpha)=0_{m}$. Тогда справедливо неравенство $[X-E] a_{0} \neq 0_{n}$ и для достаточно малых значений $a$ имеет место оценка

$$
\begin{aligned}
&\left\|x\left(\omega, a^{*}, \mu^{*}\right)-a^{*}\right\| \geqslant \\
& \geqslant \alpha \mid\left\|[X-E]\left(a_{0}+\bar{a}(\alpha)\right)\right\|-\left\|\alpha^{-1} S(\alpha)\left(p\left(a_{0}+\bar{a}(\alpha), \mu_{0}-\mu(\alpha)\right)+S^{-1}(\alpha) \varphi\left(a^{*}, \mu^{*}\right)\right)\right\|> \\
&>\frac{\alpha}{2}\left\|[X-E] a_{0}\right\|>0 .
\end{aligned}
$$

При этом $x\left(\omega, a^{*}, \mu^{*}\right) \neq a^{*}$, т.е. у системы (1)-(2) не происходит ветвления периодических решений от бесконечности.

Итак, при решении задачи о ветвлении периодического решения от бесконечности необходимо рассматривать критический случай по линейному приближению $\operatorname{det}(X-E)=0$. При этом система

$$
[X-E] a_{0}=0_{n}
$$


имеет общее решение вида $a_{0}=K z \in \operatorname{ker}[X-E]$, где $K$ - фундаментальная $(n \times r)$-матрица решений системы (3), $z \in \mathbb{R}^{r}$ - произвольный вектор. Допустим, при некотором значении $a_{0}=$ $K z_{0}$ выполняется равенство

$$
p\left(a_{0}, \mu_{0}\right)=0_{n} .
$$

Заметим, что равенство (4) необходимо для ветвления большого периодического решения с направлением $\left(a_{0}, \mu_{0}\right)$. Действительно, при условии $p\left(a_{0}, \mu_{0}\right) \neq 0_{n}$ выберем совместную параметризацию

$$
a^{*}=\alpha\left(a_{0}+\bar{a}(\alpha)\right), \quad \mu^{*}=\alpha^{-1}\left(\mu_{0}+\bar{\mu}(\alpha)\right),
$$

в которой $\alpha>0, \bar{a}(\alpha)$ и $\bar{\mu}(\alpha)$ - произвольные функции,

$$
\lim _{\alpha \rightarrow+\infty} \bar{a}(\alpha)=0_{n}, \quad \lim _{\alpha \rightarrow+\infty} \bar{\mu}(\alpha)=0_{m} .
$$

Тогда в силу равенства (3) для достаточно малых значений $\alpha$ имеет место оценка

$$
\left\|x\left(\omega, a^{*}, \mu\right)^{*}-a^{*}\right\| \geqslant\left\|S(\alpha) p\left(a_{0}+\bar{a}(\alpha), \mu_{0}+\mu(\alpha)\right)\right\|-\left\|\varphi\left(a^{*}, \mu^{*}\right)\right\|>\frac{1}{2}\left\|S(\alpha) p\left(a_{0}, \mu_{0}\right)\right\|>0 .
$$

При этом решение $x\left(\omega, a^{*}, \mu^{*}\right)$ не является периодическим.

Предположим также, что выполняется условие

$$
\operatorname{rang} J\left(a_{0}, \mu_{0}\right)=n \leqslant r+m,
$$

в котором $J\left(a_{0}, \mu_{0}\right)=\left[p_{a}^{\prime}\left(a_{0}, \mu_{0}\right) K p_{\mu}^{\prime}\left(a_{0}, \mu_{0}\right)\right]$.

Теорема 1. При условиях (3), (4), (5) система (1)-(2) имеет большое $\omega$-периодическое решение вида $x\left(t, a^{*}, \mu^{*}\right), a^{*}=\alpha\left(a_{0}+K z(\alpha)\right), \mu^{*}=\alpha^{-1}\left(\mu_{0}+\bar{\mu}(\alpha)\right)$,

$$
\lim _{\alpha \rightarrow+\infty} z(\alpha)=0_{n}, \quad \lim _{\alpha \rightarrow+\infty} \bar{\mu}(\alpha)=0_{m} .
$$

Доказательство. Пара $(a, \mu)$ определяет периодическое решение системы типа (1) тогда и только тогда, когда она удовлетворяет равенству $x(\omega, a, \mu)-a=0_{n}$ (см. [3]). Подставим в это равенство $a=a^{*}=\alpha\left(a_{0}+K z\right), \mu=\mu^{*}=\alpha^{-1}\left(\mu_{0}+\lambda\right), \alpha>0$. В силу условий (3), (4), (5) получим уравнение

$$
x\left(a^{*}, \mu^{*}\right)-a^{*}=S(\alpha)\left(J\left(a_{0}, \mu_{0}\right) \cdot:(z, \lambda)+\tilde{p}(z, \lambda)+S^{-1}(\alpha) \varphi\left(a^{*}, \mu^{*}\right)\right)=0_{n},
$$

где $\tilde{p}(z, \lambda)=p\left(a_{0}+K z, \mu_{0}+\lambda\right)-J\left(a_{0}, \mu_{0}\right) \cdot:(z, \lambda)$. По условию (5) выберем разложение $J\left(a_{0}, \mu_{0}\right) \cdot:(z, \lambda)=B \nu+B_{1} \nu_{1}$, в котором $n \times n$-матрица $B$ составлена из линейно независимых столбцов, а $(n \times(n-m-r))$-матрица $B_{1}$ составлена из остальных столбцов. Допустим, $\nu_{1}=0_{n-m-r}$. Определим функцию

$$
\psi(\alpha, \nu)=S^{-1}(\alpha) \varphi\left(a^{*}, \mu^{*}\right)+\tilde{p}(z, \lambda) .
$$

Без ограничения общности рассуждений будем предполагать, что функция $\psi(\alpha, \nu)$ при $\alpha \rightarrow+\infty$ и $\nu \rightarrow 0_{n}$ доопределена условиями $\psi\left(+\infty, 0_{n}\right)=0_{n}, \psi_{\nu}^{\prime}\left(+\infty, 0_{n}\right)=0_{n n}$. Тогда уравнение (6) примет вид

$$
g(a, \nu)=B \nu+\psi(\alpha, \nu)=0_{n}
$$

Так как $g\left(+\infty, 0_{n}\right)=0_{n}, g_{\nu}^{\prime}\left(+\infty, 0_{n}\right)=B$ и $\operatorname{det} B \neq 0$, то по теореме о неявной функции уравнение (7) определяет функцию $\nu=\nu(\alpha), \Delta<\alpha, \nu(+\infty)=0_{n}$, для которой $g(\alpha, \nu(\alpha)) \equiv 0_{n}$. Зная $\nu=\nu(\alpha)$ и учитывая, что $\nu_{1}=0_{n-m-r}$, получим функции $z=z(\alpha), \lambda=\lambda(\alpha)$ и пару $\left(\alpha^{*}, \mu^{*}\right)$, в которой

$$
a^{*}=\alpha\left(a_{0}+K z\right), \quad \mu^{*}=\alpha^{-1}\left(\mu_{0}+\lambda(\alpha)\right) .
$$

Так как найденная пара удовлетворяет уравнению $(6)$ и $\left(\alpha^{*}, \mu^{*}\right) \rightarrow\left(\infty, 0_{m}\right)$ при $\alpha \rightarrow+\infty$, то решение $x\left(t, a^{*}, \mu^{*}\right)$ системы (1)-(2) - большое $\omega$-периодическое решение по определению. Теорема 1 доказана. 
Лемма 1. Если выполняются условия теоремы 1 и при всех больших $\alpha>0$ справедливо неравенство

$$
\left\|X+\alpha^{-1} S(\alpha) p_{a}^{\prime}\left(a_{0}, \mu_{0}\right)\right\| \leqslant 1-b h(\alpha),
$$

где $b>0$-некоторое число, $h(\alpha)>0, h(+\infty)=0$, то большое $\omega$-периодическое решение $x\left(t, a^{*}, \mu^{*}\right)$ системы (1)- (2) асимптотически устойчиво.

Доказательство. Для решения $x\left(t, a^{*}, \mu^{*}\right)$ составим систему в вариациях. Матрица монодромии этой системы по условию (2) имеет вид

$$
x_{a}^{\prime}\left(\omega, a^{*}, \mu^{*}\right)=X+p_{a}^{\prime}\left(a^{*}, \mu^{*}\right)+\varphi_{a}^{\prime}\left(a^{*}, \mu^{*}\right)=X+\alpha^{-1} S(\alpha) p_{a}^{\prime}\left(a_{0}, \mu_{0}\right)+W(\alpha),
$$

где матрица

обладает свойством

$$
W(\alpha)=p_{a}^{\prime}\left(a^{*}, \mu^{*}\right)+\varphi_{a}^{\prime}\left(a^{*}, \mu^{*}\right)-\alpha^{-1} S(\alpha) p_{a}^{\prime}\left(a_{0}, \mu_{0}\right)
$$

$$
\lim _{\alpha \rightarrow+\infty} \alpha S^{-1}(\alpha) W(\alpha)=0_{n n}
$$

По условию при всех достаточно больших $\alpha>0$ имеем

$$
\left\|x_{a}^{\prime}\left(\omega, a^{*}, \mu^{*}\right)\right\| \leqslant\left\|X+\alpha^{-1} S(\alpha) p_{a}^{\prime}\left(a_{0}, \mu_{0}\right)\right\|+\|W(\alpha)\| \leqslant 1-\frac{b h(\alpha)}{2} .
$$

При этом спектральный радиус матрицы монодромии $x_{a}^{\prime}\left(\omega, a^{*}, \mu^{*}\right)$ удовлетворяет оценке

$$
\rho\left(x_{a}^{\prime}\left(\omega, a^{*}, \mu^{*}\right)\right) \leqslant\left\|x_{a}^{\prime}\left(\omega, a^{*}, \mu^{*}\right)\right\|<1 .
$$

Значит, решение $x\left(\omega, a^{*}, \mu^{*}\right)$ асимптотически устойчиво по линейному приближению. Лемма доказана.

Проверка необходимых условий наличия большого периодического решения путем поиска направления ветвления предполагает проверку равенств (3), (4). С вычислительной точки зрения это может представлять затруднения. Теорему 1 целесообразно дополнить признаком, опирающимся на проверку условий типа неравенств. С этой целью вместо необходимых условий типа равенства для направлений ветвления выберем априорную оценку начального значения решения.

Допустим, в окрестности бесконечности левый оператор монодромии имеет вид

$$
x(-\omega, a, \mu)=\bar{X} a+\bar{p}(a, \mu)+\bar{\varphi}(a, \mu),
$$

где $\bar{X}=X(-\omega), \bar{p}(\alpha a, \mu / \alpha)=S(\alpha) \bar{p}(a, \mu), \lim _{\alpha \rightarrow+\infty} \alpha S^{-1}(\alpha) \bar{\varphi}(\alpha a, \mu / \alpha) \equiv 0_{n}$.

Применив формулу Эйлера для однородной функции, получим соотношения:

$$
\begin{gathered}
\bar{P}(a) a=\bar{p}\left(a, 0_{m}\right), \quad \bar{P}(a)=\operatorname{diag}\left(\frac{1}{k_{1}}, \ldots, \frac{1}{k_{n}}\right) \bar{p}_{a}^{\prime}\left(a, 0_{m}\right), \\
P(a, \mu) a=p(a, \mu), \quad P(a, \mu)=\operatorname{diag}\left(\frac{1}{k_{1}}, \ldots, \frac{1}{k_{n}}\right) p_{a}^{\prime}(a, \mu) .
\end{gathered}
$$

Будем предполагать, что неравенства для векторов и матриц имеют покомпонентный смысл. Определим подмножества конуса неотрицательных векторов

$$
K(\sigma)=\left\{a \in R^{m}:\|a\|>\sigma, a \geqslant 0_{m}\right\}, \quad \bar{K}(\sigma)=\left\{a \in R^{m}:\|a\|=\sigma, a \geqslant 0_{m}\right\} .
$$

Допустим, что при всех $\lambda \in K(1)$ выполняются условия

$$
X \geqslant 0_{n n}, \quad \bar{X} \geqslant 0_{n n}
$$

и при всех больших $\alpha>0$ для некоторого значения $\mu=\mu_{0}$ справедливы оценки

$$
\begin{gathered}
\bar{P}(\lambda) \geqslant B \geqslant 0_{n n}, \quad \bar{X}+B>0_{n n}, \\
\left\|X+\alpha^{-1} S(\alpha) \bar{P}(\lambda)\right\| \leqslant 1-\frac{b}{\alpha^{1+k}}, \quad b>0, \quad k>0, \\
P\left(\lambda, \mu_{0}\right) \geqslant D \geqslant 0_{n n}, \quad X+D>0_{n n}, \\
\left\|X+\alpha^{-1} S(\alpha) P\left(\lambda, \mu_{0}\right)\right\| \leqslant 1-d / \alpha^{1+k}, \quad d>0, \quad k>0 .
\end{gathered}
$$




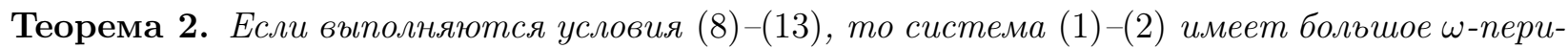
одическое решение.

Доказательство. В силу неравенств (9), (12), (13) по групповому свойству решений существует такое число $\sigma>0$, что $x(\omega, a, \mu) \in K(\sigma)$ при условиях $a \in K(\sigma)$ и $\mu=\mu_{0} / \alpha, \alpha>\sigma$, т.е. множество $K(\sigma)$ инвариантно для правого оператора монодромии.

Так как $\lim _{\alpha \rightarrow+\infty} \alpha S^{-1}(\alpha) \bar{\varphi}(\alpha a, \mu / \alpha) \equiv 0_{n}$, то существует такое $\delta_{1} \geqslant \sigma$, что $\left\|\bar{\varphi}\left(\alpha \lambda, 0_{m}\right)\right\| \leqslant b /\left(2 \alpha^{k}\right)$ для любого $a=\alpha \lambda \in K\left(\delta_{1}\right)$. При этом из условия (11) следует оценка

$$
\|x(-\omega, a, 0)\|<\left(1-\frac{b}{2\|a\|^{k}}\right)\|a\|<\|a\| .
$$

Поэтому найдется такое $q>2$, что при достаточно большом $\delta_{2} \geqslant \delta_{1}$ для любого $\alpha>\delta_{2}$ справедлива оценка

$$
\left\|x\left(-\omega, a \lambda, \frac{\mu_{0}}{\alpha}\right)\right\|<\left(1-\frac{b}{q \alpha^{k}}\right) \alpha<\alpha .
$$

Тогда по групповому свойству решений получим, что $\left\|x\left(\omega, \alpha \lambda, \mu_{0} / \alpha\right)\right\|>\alpha$.

Рассуждая аналогично предыдущему, можно установить, что условию (13) при достаточно большом $\delta_{3} \geqslant \sigma$ для любого $\alpha>\delta_{3}$ справедлива оценка

$$
\left\|x\left(\omega, \alpha \lambda, \frac{\mu_{0}}{\alpha}\right)\right\|<\left(1-\frac{d}{2 \alpha^{k}}\right) \alpha<\alpha .
$$

Итак, при любом $\alpha>\max \left\{\delta_{2}, \delta_{3}\right\}$ существует конусный отрезок

$$
K_{\alpha}=\left\{a=\alpha \lambda \in \mathbb{R}^{n}: 0<\Delta(\alpha) \leqslant \alpha \leqslant \delta(\alpha), \lambda \geqslant 0_{n},\|\lambda\|=1\right\},
$$

для которого $\lim _{\alpha \rightarrow+\infty} \Delta(\alpha)=+\infty$ и

$$
x\left(\omega, \alpha \lambda, \mu_{0} / \alpha\right): K_{\alpha} \rightarrow \operatorname{int} K_{\alpha} \quad \text { или } \quad x\left(-\omega, \alpha \lambda, \mu_{0} / \alpha\right): K_{\alpha} \rightarrow \operatorname{int} K_{\alpha} .
$$

Так компактное множество $K_{\alpha}$ гомеоморфно выпуклому, например, множеству

$$
\left\{a \in \mathbb{R}^{n}: \Delta(\alpha) \leqslant\|a\|_{1} \leqslant \delta(\alpha), a \geqslant 0_{n},\|a\|_{1}=\sum_{i=1}^{n}\left|a_{i}\right|\right\},
$$

то по теореме Брауэра о неподвижной точке существует значение $a^{*}=a(\alpha) \in \operatorname{int} K_{\alpha}$, причем $\lim _{\alpha \rightarrow+\infty} \alpha^{*}=+\infty$. Итак, $x\left(t, \alpha^{*}, \mu^{*}\right)$ - большое $\omega$-периодическое решение для системы $(1)-(2)$ по определению, где $\mu^{*}=\mu_{0} / \alpha$. Теорема 2 доказана.

Пример. Рассмотрим дифференциальное уравнение

$$
\dot{x}=\frac{3 \cos t}{2+\sin t} x+g(t, x, \mu), \quad g(t, x, \mu)=c_{1} \mu x^{4 / 3}+\frac{1}{8}(2+\sin )^{3} c_{2} x^{1 / 3} .
$$

Так как $g(t, \alpha x, \mu / \alpha)=\alpha^{1 / 3} g(t, x, \mu)$, то уравнение (14) можно рассматривать в качестве системы вида (1) порядка $n=1$ с периодом $2 \pi$ по $t$. При этом вычислим $X(t)=(2+\sin t)^{3} / 8, X=1$,

$$
p(a, \mu)=X \int_{0}^{2 \pi} X^{-1}(t) g(t, X(t) a, \mu) d t=2 \pi\left(c_{1} \mu a^{4 / 3}+c_{2} a^{1 / 3}\right) .
$$

Условие (3) выполняется при любом $a_{0}$. Для определенности выберем $a_{0}=1$. Тогда условие (4) выполняется при $\mu_{0}=-c_{2} / c_{1}$. При этом получим, что $p_{a}^{\prime}\left(a_{0}, \mu_{0}\right)=-2 \pi c_{2}, p_{\mu}^{\prime}\left(a_{0}, \mu_{0}\right)=-2 \pi c_{1}$, $\operatorname{rang} J\left(a_{0}, \mu_{0}\right)=1$, т.е. справедливо условие (5). Итак, по теореме 1 уравнение (14) имеет большое $2 \pi$-периодическое решение с направлением ветвления $\left(1,-c_{2} / c_{1}\right)$. При $c_{2}>0$ для всех достаточно больших значений $\alpha>0$ справедливо неравенство $X+\alpha^{-1} S(\alpha) p_{a}^{\prime}\left(a_{0}, \mu_{0}\right)=1-2 \pi c_{2} \alpha^{-2 / 3}<1$. Тогда по лемме большое $2 \pi$-периодическое решение асимптотически устойчиво. Полученные выводы согласуются с фактом существования решения $x\left(t, a^{*}, \mu^{*}\right)=(2+\sin t)^{3} a^{*} / 8, a^{*}=\alpha$ уравнения (14) при $\mu=\mu^{*}=-c_{2} \alpha / c_{1}$. 


\section{СПИСОК ЛИТЕРАТУРЫ}

1. Абрамов В. В. Устойчивость малого периодического решения// Вестн. РАЕН. - 2013. - 13, № 4. C. $3-5$.

2. Абрамов B. В. Ветвление периодического решения с положительным начальным значением// Вестн. PAEH. - 2017. - 17, № 4. - C. 4-7.

3. Красносельский M. А. Оператор сдвига по траекториям дифференциальных уравнений. - М.: Наука, 1966.

Абрамов Владимир Викторович

Рязанский государственный университет имени С. А. Есенина

E-mail: v.abramov@365.rsu.edu.ru 